\title{
Migrantes latinoamericanos más allá de la frontera norte
}

\section{Carlos Véjar Pérez-Rubio'}

Recepción: 12 de diciembre de 2014 / Aprobación: 29 de enero de 2015

\section{Resumen}

El tema de los migrantes latinoamericanos en Estados Unidos y Canadá es analizado en este ensayo partiendo del concepto antropológico de los viajes, que han estado asociados siempre al género humano, en todas las culturas y ámbitos geográficos, desde los nómadas de la prehistoria hasta los turistas de la actualidad. Se advierte en el análisis que este fenómeno ha sido crucial para el desarrollo de las diversas civilizaciones, que se han forjado en buena medida con el tránsito e incorporación de individuos y grupos ajenos, si bien muchas veces con problemas, como es el caso hoy en día de la masiva corriente de migrantes que pasan de los países subdesarrollados a los desarrollados en busca de condiciones adecuadas para una vida digna, inaccesible en sus países de origen. Los millones de trabajadores migrantes de Latinoamérica que se encuentran en Estados Unidos y Canadá son un buen ejemplo de ello.

\section{Palabras clave}

Migrantes; viajes; identidad; cultura; ambiente

\section{Abstract}

In this essay, the theme of Latin American migration in the United States and Canada is analyzed based on the anthropological concept of travel. From prehistory nomads to today's tourists, this concept has majorly been concomitant to human nature in all cultures and geographical areas. Throughout this analysis it is noted that this phenomenon has crucially contributed to the development of diverse civilizations largely built up by the influence of foreign individuals and groups either in transit or permanently settled in. Reportedly, these individuals and groups often have problems in their countries, such as in the case of today's massive outflow of migrants from undeveloped to developed countries seeking suitable conditions for a life in dignity, unattainable in their countries of origin. The millions of Latin American migrant workers who live in the United States and Canada clearly exemplify such situation.

1 Mexicano. Arquitecto, Maestro en Historia del Arte y Doctor en Estudios Latinaméricanos por la Universidad Nacional Autónoma de México (UNAM). Investigador del Centro de Investigaciones sobre América Latina y el Caribe (CIALC) y profesor de la Facultad de Arquitectura, ambos en la UNAM. Correo electrónico: elaleph@archipielago.com.mx 


\section{Key words}

Migrants; travel; identity; culture; environment

\section{Resumo}

O tópico dos migrantes latino-americanos nos Estados Unidos e o Canadá é analisado neste trabalho com base no conceito antropológico das viagens que têm sido associados sempre ao gênero humano, em todas as culturas e ambientes geográficos, dos nômades da pré-história até os turistas do tempo presente. É notado na análise que este fenômeno foi crucial para o desenvolvimento das civilizações diversas que forjaram em grande parte com o tráfego ea inclusão de indivíduos e grupos externos, embora muitas vezes com problemas, como é o caso hoje dia do fluxo maciço de migrantes que se deslocam de países em desenvolvimento para países desenvolvidos à procura de condições adequadas para uma vida digna, inacessível em seus países de origem. Os milhões de trabalhadores migrantes da América Latina que estão nos Estados Unidos e o Canadá são um bom exemplo.

\section{Palavras chave}

Migrantes; viajar; identidade; cultura; ambiente

\section{Los viajeros}

os viajes han estado asociados siempre al género humano, en todas las culturas y en todos los ámbitos geográficos. Desde la óptica de los antropólogos, el hombre primitivo era un constante viajero, un nómada que recorría los territorios a su alcance en busca del alimento que le proporcionaban la caza, la pesca y la recolección de frutos. El surgimiento de las primeras civilizaciones a partir del descubrimiento de la agricultura y la domesticación de animales propició, es cierto, el sedentarismo en los seres humanos, más no la cancelación de los viajes.

Por el contrario, el desarrollo de las fuerzas productivas y de la división social del trabajo estimuló pronto el intercambio entre los pueblos, realizado a través de viajes en los más variados medios de transporte. Surgen también entonces diversos tipos de viajeros, según los motivos o encomiendas que los impulsan. De algunos de ellos darán cuenta la historia, la leyenda y la fantasía. La literatura universal se nutre de todos estos elementos. La Odisea, de Homero, es uno de los primeros ejemplos de ello en la cultura occidental. En la época moderna, podemos mencionar el libro del escritor irlandés Laurence Sterne, Viaje sentimental por Francia e Italia, publicado en 1768; el de Alejandro de Humboldt, Ensayo político sobre el reino de la Nueva España, basado en el viaje realizado por el intelectual alemán en el México de 18031804; y La vida en México durante una residencia de dos años en ese país, 
escrito en 1843 por Frances Erskine Inglis, más conocida como Madame Calderón de la Barca, esposa del primer Embajador de España en México después de la independencia.

Otros dos célebres viajeros de aquellos tiempos son el estadunidense John L. Stephens y el inglés Frederick Catherwood, conocidos como los primeros mayistas (García Moll, 1996, pp. 51-52), quienes realizaron en sus viajes por el mundo maya descubrimientos asombrosos que dejaron plasmados en sus libros Incidents of Travel in Central América, Chiapas and Yucatán, de 1841; e Incidents of travel in Yucatán, de 1843, los cuales han sido punto de partida para muchos investigadores, al estar escritos sin las fantasías propias de los viajeros del siglo XIX.

La figura del viajero se vuelve clave en el desarrollo de las civilizaciones, que se enriquecen con el transitar de individuos y de grupos, de costumbres y de ideas. Nuestra América es protagonista de ello desde tiempos inmemoriales, de Mesoamérica al Tahuantinsuyo. Un viajero que, con el paso del tiempo, incluirá entre sus filas al que busca principalmente solaz, descanso, esparcimiento, conocimientos y aventura, esto es, al hoy llamado turista.

El turismo ${ }^{2}$, en la medida en que significa un desplazamiento hacia el mundo del Otro, deviene una experiencia cultural. Este fenómeno, proyectado masivamente por el desarrollo económico y tecnológico del mundo moderno y sus repercusiones en los hábitos, costumbres y patrones de consumo de la sociedad, se ha convertido hoy en día en un sector fundamental de la economía y la cultura de América Latina y el Caribe. La simbiosis entre el patrimonio natural y el cultural ha hecho surgir en la región una pujante industria turística, que en algunos casos es verdadero motor del desarrollo. Los productos con los que se cuenta son ricos y variados: centros históricos de ciudades, monumentos y sitios declarados por la UNESCO Patrimonio Mundial de la Humanidad; museos, galerías y exposiciones; arqueoturismo (aéreo, terrestre y subacuático); festivales y ciclos de teatro, cine, música y danza; fiestas tradicionales, folclore y cultura popular; creación artística y artesanal; etnoecoturismo; turismo comunitario; turismo gastronómico; enoturismo; turismo de montaña; turismo de sol y playa, y sus componentes culturales: turismo náutico, turismo deportivo, turismo de aventura, etcétera.

Si bien es cierto que su masificación se logró a partir de la creación de los grandes centros vacacionales en la segunda mitad del siglo XX, hoy ha cobrado impulso un modelo alternativo a partir de los viajes que propician el conocimiento y el goce de la diversidad cultural. Si pensamos que la sociedad industrializada avanza, en mayor o menor grado, hacia el hastío, la indiferencia y la monotonía - conquistas indiscutibles del "desarrollo"-el turismo parece ser un medio idóneo para que las personas se evadan de esa realidad y encuentren en

2 La palabra "turismo" proviene del vocablo inglés "tour" que significa viaje y del vocablo francés "tour" que significa asimismo viaje o excursión. El origen de ambos vocablos es el latín "tornus". 
otras latitudes la aventura que las apasione con más intensidad que los programas de su televisión, el internet, las redes sociales o las películas de moda. Otro tipo de descanso y diversión. A la expectativa de las bellezas físico-ambientales desean sumar ahora el conocimiento del medio cultural del sitio de destino. La experiencia simbólica del viaje se opone así al frívolo y masificado flujo turístico que deslava identidades y enajena más aún al ser humano de nuestro tiempo. El turista vuelve a sentirse viajero y, por tanto, disfruta de nuevo la aventura. El patrimonio cultural tangible e intangible, la mayor riqueza con que cuentan nuestros pueblos y base indiscutible de su identidad, cobra así importancia fundamental y se convierte en piedra de toque para que puedan alcanzar su dignidad, bienestar e independencia.

\section{Los migrantes}

Pero hay también otro tipo de viajeros. Elaborar un concepto cultural de América Latina y el Caribe que desborde los estrechos límites de las fronteras geopolíticas, para incluir a la parte de su población asentada en otras latitudes, implica reconocer al fenómeno migratorio como uno de los que más impactan actualmente a las estructuras económicas, sociales, políticas y culturales de la región. Bolivianos y paraguayos en Buenos Aires; ecuatorianos, colombianos y peruanos en Madrid; haitianos, dominicanos y puertorriqueños en Nueva York; cubanos en Miami; nicaragüenses en Costa Rica; chilenos, argentinos y uruguayos en México; mexicanos y centroamericanos en California, Texas, Illinois... en Canadá. Campesinos en las labores agrícolas, trabajadores en los servicios domésticos y comerciales, asilados políticos, estudiantes universitarios y profesionales de las más diversas disciplinas, empresarios, funcionarios, proletarios... La variedad es asombrosa. Las identidades se deslavan, se transforman, se modifican. El idioma, la cultura, la religión, la alimentación, las costumbres, las tradiciones, los principios, los valores, todo ello se resiente, se sacude. Carlos Drummond de Andrade (1902-1987), considerado uno de los mayores poetas brasileños, lo expresa así en su poema "La ilusión del emigrante":

Cuando vine de mi tierra

si es que vine de mi tierra

(¿no estoy muerto por allá?),

la corriente del río

me susurró vagamente

que debía quedarme

allá donde me despedía.

Los cerros, al palidecer

en el entrecerrarse de la tarde,

parecían decirme

que ya no se puede volver, 
Cuando vine, si es que vine de algún lugar para otro

el mundo giraba, ajeno

a mi deslucida persona, y en sus giros entreví que no se va ni se vuelve de sitio alguno o ninguno

Que cargamos las cosas, moldura de nuestra vida, cerco rígido de alambre, en la más anónima célula, y un suelo, una risa, una voz resuenan incesantemente en nuestras hondas paredes.

Nuevas cosas, sucediéndose, eluden nuestra hambre del primitivo alimento.

Las revelaciones son máscaras de lo real más oscuro, esa herida esparciéndose en la piel de nuestras almas.

Cuando vine de mi tierra, no vine, me perdí en el espacio, en la ilusión de haber salido. Ay de mí, no salí nunca. Allá estoy yo, enterrado, debajo de mansas voces. debajo de sombras negras, debajo de minas de oro, debajo de generaciones, debajo, yo lo sé, de mí mismo, este viviente engañado y engañoso ${ }^{3}$.

Esto es en cierta forma el problema del exilio, palabra antigua en nuestra lengua, reseñada ya en el siglo XIII, cuando aparece en textos de Gonzalo de Berceo y Alfonso el Sabio. De origen latino, la palabra era usada en la cultura romana bajo la forma exsilum, cuyo significado etimológico era "saltar afuera". La palabra afín más usual es destierro.

Si bien las migraciones y lo que ellas conllevan, como decíamos, son inherentes al devenir del género humano, hoy en día se han convertido en uno de los retos más grandes que tiene que afrontar el mundo globalizado. Desde hace varias décadas, el flujo migratorio de los países pobres a los países ricos

3 Este poema fue publicado en una de sus obras póstumas: Farewell (1996). La traducción es del poeta uruguayo Saúl Ibargoyen y apareció publicada en el número 8 de Archipiélago. Revista Cultural de Nuestra América, septiembre-octubre, 1996, p. 1. 
se disparó notablemente, debido en gran medida a los ajustes neoliberales realizados a la economía mundial, que acentuaron las desigualdades sociales y polarizaron más aún al planeta.

Para hablar de México, baste decir que este país se convirtió desde el 2004 en el principal expulsor de inmigrantes del mundo, por encima incluso de China. El destino, en la mayoría de los casos, es Estados Unidos. Estudios recientes indican que cada año 485 mil mexicanos se ven obligados a abandonar su país para buscar trabajo en el vecino del norte, lo que significa un incremento de 400\% en los últimos 25 años (Tépach Marcial, 2012)4.

El mismo destino estadunidense lo comparten la mayor parte de los migrantes de muchos otros países latinoamericanos, particularmente, los del área de Centroamérica y el Caribe. Según datos de la Oficina del Censo estadounidense, a finales de 2007 la población de origen hispano y latino en ese país estaba constituida en primer lugar, por mexicanos (63.0\%); en segundo lugar, por puertorriqueños (9.2\%); en tercer lugar, por cubanos (3.5\%); en cuarto lugar, por salvadoreños (3.2\%); en quinto lugar, por dominicanos (2.8\%); y el 16.3\% restante por migrantes del resto del subcontinente (Ennis, Ríos-Vargas y Albert, 2012). El Pew Hispanic Center (PHC) determinó en 2011 que los latinos e hispanos constituyen la primera minoría del país, con cerca de 52 millones de personas (Pew Research Center, s.f.) -17\% de la población total estadunidense-, cantidad que para el año 2050 se triplicará, lo que representará un 29\% de la población. De ellos, más de 12 millones son indocumentados, dos terceras partes de los cuales son de origen mexicano. En su inmensa mayoría pertenecen a esa diáspora de trabajadores latinoamericanos y caribeños, que han tenido que recurrir a esta acción extrema para encontrar el medio de subsistencia que en sus países de origen se les niega o dificulta.

Este proceso migratorio, en el que anida la contradicción no resuelta entre mundialización y universalismo, por un lado, y singularidad y particularismo, por el otro, ha exacerbado fenómenos tales como la hibridación y la transculturación, la intolerancia, el racismo y la xenofobia, cuyas manifestaciones violentas y crueles son un impedimento para cualquier convivencia civilizada y una amenaza para la paz mundial. El levantamiento de un muro en la frontera de México y Estados Unidos, el incremento de las deportaciones masivas y las barreras impuestas por los republicanos y el Tea Party a la reforma migratoria de Obama en el Congreso, son tan solo algunas pruebas fehacientes de ello.

El caso de Canadá es muy diferente. Se estima que hoy en día viven allí alrededor de 100 mil mexicanos, quienes conforman la fracción más grande de latinoamericanos en dicho país, cuyo total se estima en alrededor de 400 mil. La población total de la nación canadiense es de poco más de 35 millones de habitantes, quienes se distribuyen en un territorio de casi 10 millones de

4 De 1911 a 1930 emigraron 678291 mexicanos a los Estados Unidos (González Casanova, 1969). 
kilómetros cuadrados, es decir, es el segundo país más grande del planeta, superado tan solo por Rusia. Esta migración hispana o latina es relativamente reciente y, a diferencia de la que va a Estados Unidos, procede en su mayor parte de la clase media, con los inmigrantes calificados superando a los no calificados, lo cual la hace menos conflictiva y contribuye a enriquecer la diversidad de la población canadiense, en la que han dejado su huella las migraciones de las más variadas latitudes. No viven en enclaves segregados, en su mayor parte están legalizados, es decir, tienen residencia o están incluso nacionalizados canadienses, lo cual facilita su integración al entorno social.

Los pueblos latinoamericanos y caribeños, cuya identidad encuentra su raíz más profunda en las migraciones que desde época remota han dejado huella en su territorio, y en el mestizaje étnico-cultural que las ha acompañado, no pueden ni deben enfrentar esta situación aislados. Es por ello alentador el impacto de las manifestaciones multitudinarias realizadas en múltiples ciudades estadounidenses por sus comunidades migrantes -hispanos y latinos- el 11 de abril y el 1 de mayo de 2006, en las que ondearon orgullosas las banderas mexicanas, acompañadas de las ecuatorianas, las colombianas, las salvadoreñas, las peruanas, las bolivianas, las puertorriqueñas, las dominicanas y muchas otras más. Una interesante e inédita muestra de unidad en torno a sus justas demandas, que sentó las bases para ulteriores movilizaciones, incluida su participación en 2011 en el movimiento Occupy Wall Street, el distrito financiero de Nueva York, concentración de protesta contra el poder omnímodo de las empresas transnacionales y las evasiones fiscales sistemáticas del 1\% más rico de la población. Su voto, por cierto, fue fundamental para el triunfo del candidato demócrata, Barack Obama, en las elecciones presidenciales del 6 de noviembre de 2008.

"Somos una nación de inmigrantes y una nación de leyes", dijo el congresista estadounidense Jim Sensenbrenner, autor de la polémica ley anti-inmigrante que ha polarizado a Estados Unidos. Pero eso es falso. Las primeras oleadas de europeos que llegaron a estas tierras americanas, anglosajones mayoritariamente, no eran inmigrantes, sino colonizadores. Estados Unidos no es una nación de inmigrantes, es un asentamiento colonialista preponderantemente blanco, wasp (white, anglo, saxon, protestant). Los colonizadores se apoderaron de la tierra por la fuerza, exterminaron a la mayor parte de la población nativa "no blanca" y marginaron a los sobrevivientes en reservaciones estilo apartheid, que subsisten hasta la fecha. Desde 1790, el gobierno estadounidense estableció que el derecho a ser ciudadano naturalizado se reservaba a las "personas blancas libres" (free white persons) (Imai, 19 de marzo de 2013) $)^{5}$. Así permaneció la norma hasta 1952. Las leyes migratorias de este país siempre han justificado el trato discriminatorio contra los que consideran seres

$5 \quad$ Conocida alternativamente como el Acta de la Nacionalidad, el Acta de Naturalización de 1790 restringía la ciudadanía para los extranjeros que fueran a free white person, y que hubieran estado en los Estados Unidos por dos años. 
de otros mundos, extraños, distintos, aliens, sean latinoamericanos-caribeños, asiáticos o africanos. Son leyes racistas.

Hoy, en la segunda década del siglo XXI, la situación de nuestros migrantes más allá de la frontera norte, en Estados Unidos principalmente, es complicada. Todos ellos han resentido los efectos de la crisis económica mundial, que los ha obligado a apretarse el cinturón y disminuir sus remesas a sus países de origen, que dependen en gran medida de ellas para su precario desarrollo. La crispación social, la inestabilidad y la inseguridad son evidentes y se manifiestan en todos los órdenes. Esperemos que la composición multirracial y pluricultural del gran país del norte americano restaure pronto sus cuarteaduras y que se establezcan patrones de convivencia basados no solamente en el consumo y todo lo que ello significa, sino en la solidaridad social, la participación democrática, la libertad responsable y el respeto para todos. $Y$ que los latinoamericanos y caribeños encuentren en esas latitudes las mejores condiciones para su desenvolvimiento, conscientes de que conforman en ellas una extensión cultural y moral de la patria grande.

Nuestros migrantes. Es necesario definir acciones que contribuyan a reafirmar la identidad cultural, el sentido de pertenencia y el orgullo de ser de los latinoamericanos y caribeños residentes en las más variadas latitudes, basadas en sus raíces, tradiciones y luchas ancestrales, así como estimular en ellos la conciencia de unidad, indispensable para lograr la satisfacción de sus demandas y mejores condiciones de vida. Habiten donde habiten, se encuentren donde se encuentren, todos ellos estarán residiendo, culturalmente, en un confín latinoamericano.

\section{Referencias}

Calderón de la Barca, Madame. (1974). La vida en México durante una residencia de dos años en ese país. México: Editorial Porrúa.

Drummond de Andrade, Carlos. (septiembre-octubre, 1996). Farewell (traductor Saúl Ibargoyen). Archipiélago. Revista Cultural de Nuestra América, (8).

Ennis, Sharon R.; Ríos-Vargas, Merarys \& Albert, Nora G. (2012). La Población Hispana: 2010. Información del Censo del 2010. U.S. Census Bureau. Recuperado de https://www.census.gov/prod/cen2010/briefs/c2010br-04sp.pdf

García Moll, Roberto. (septiembre-octubre, 1996). Los primeros mayistas. John L. Stephens y Frederick Catherwood. Archipiélago. Revista Cultural de Nuestra América, (8).

González Casanova, Pablo. (1969). La democracia en México. México: Era.

24 Homero. (1984). La Odisea. México: Editores Mexicanos Unidos. 
Humboldt, Alejandro de. (1941). Ensayo político sobre el reino de la Nueva España. México: Editorial Pedro Robredo.

Imai, Shiho. (19 de mazo de 2013). Naturalization Act of 1790. Densho Encyclopedia. Recuperado de http://encyclopedia.densho.org/Naturalization\%20 Act\%20of\%201790/

Pew Research Center. (s.f.). Hispanics. Recuperado de http://www.pewresearch.org/data-trend/society-and-demographics/hispanics/

Stephens, John L. Ilustraciones de Catherwood, Frederick. (2003). Incidentes del viaje a Yucatán I. Madrid: Editorial Dastin.

Stephens, John L. Ilustraciones de Catherwood, Frederick. ([1841] 2010). Incidents of Travel in Central America, Chiapas and Yucatán (Vols. 1 \& 2). Cambridge: Cambridge University Press.

Sterne, Laurence. (1987). Viaje sentimental por Francia e Italia. México: Fondo de Cultura Económica.

Tépach Marcial, Reyes. (2012). El flujo migratorio internacional de México hacia los Estados Unidos de América y la captación de las remesas familiares en nuestro país, 2007-2012. Dirección general de servicios de documentación, información y análisis. Recuperado de http://www.diputados.gob.mx/ sedia/sia/se/SAE-ISS-16-12.pdf 